\title{
A quasicontinuum methodology for multiscale analyses of discrete microstructural models
}

\author{
L. A. A. Beex, R. H. J. Peerlings, ${ }^{* \dagger}$ and M. G. D. Geers \\ Department of Mechanical Engineering, Eindhoven University of Technology, P.O. Box 513, \\ 5600 MB Eindhoven, The Netherlands
}

\begin{abstract}
SUMMARY
Many studies in different research fields use lattice models to investigate the mechanical behavior of materials. Full lattice calculations are often performed to determine the influence of localized microscale phenomena on large-scale responses but they are usually computationally expensive. In this study the quasicontinuum (QC) method (Phil. Mag. A 1996; 73:1529-1563) is extended towards lattice models that employ discrete elements, such as trusses and beams. The QC method is a multiscale approach that uses a triangulation to interpolate the lattice model in regions with small fluctuations in the deformation field, while in regions of high interest the exact lattice model is obtained by refining the triangulation to the internal spacing of the lattice. Interpolation ensures that the number of unknowns is reduced while summation ensures that only a selective part of the underlying lattice model must be visited to construct the governing equations. As the QC method has so far only been applied to atomic lattice models, the existing summation procedures have been revisited for structural lattice models containing discrete elements. This has led to a new QC method that makes use of the characteristic structure of the considered truss network. The proposed QC method is, to the best of the authors' knowledge, the only QC method that does not need any correction at the interface between the interpolated and the fully resolved region and at the same time gives exact results unlike the cluster QC methods. In its present formulation, the proposed QC method can only be used for lattice models containing nearest neighbor interactions, but with some minor adaptations it can also be used for lattices with next-nearest neighbor interactions such as atomic lattices. Copyright (C) 2011 John Wiley \& Sons, Ltd.
\end{abstract}

Received 12 October 2010; Revised 21 December 2010; Accepted 21 December 2010

KEY WORDS: quasicontinuum method; multiscale; lattice model; network; discrete elements; fiber; heterogeneous materials

\section{INTRODUCTION}

A vast amount of structural lattice models (using springs, trusses, or beams) have been successfully developed for discrete microstructures of fibrous and heterogeneous materials. These conceptually simple models are able to accurately describe complex mechanisms in these materials, caused by the discrete character of their microstructures. A major advantage of lattice models is the fact that they intrinsically incorporate the discreteness of microscale phenomena, while continuum models often have to be complex or extensive to (partially) include them. Especially localized phenomena as for instance fiber failure and bond failure can be adequately captured with lattice models.

The variety of lattice models for fibrous materials and structures ranges from cardboard packages and woven structures for aerospace industry to collagen networks and other fibrous biological

\footnotetext{
${ }^{*}$ Correspondence to: R. H. J. Peerlings, Department of Mechanical Engineering, Eindhoven University of Technology, P.O. Box 513, 5600 MB Eindhoven, The Netherlands.

†E-mail: r.h.j.peerlings@tue.nl
} 
materials. Recent examples are the lattice models of Heyden [1]; Arnoux et al. [2]; Bronkhorst [3]; Clyne et al. [4]; Potluri and Manan [5]; Lomov et al. [6]; Ben Boubaker et al. [7]; Stylianopoulos and Barocas [8]; Zohdi [9]; and Hatami-Marbini and Picu [10]. For heterogeneous materials which do not consist of a discrete network at the microscale, a discrete representation containing trusses and beams is less natural, but it can still be physically relevant. This has been shown for instance by Lilliu and Van Mier [11] and Cusatis et al. [12] for concrete, by Ostoja-Starzewski and Wang [13] for epoxy plates, by Rinaldi et al. [14] for polycrystalline microstructures, and by Kim and Buttlar [15] for asphalt concrete. An overview of different structural lattice models is presented by Ostoja-Starzewski [16].

Although lattice models have the advantage that they naturally resolve microscale phenomena in discrete microstructures, their use can be computationally expensive. Lilliu and Van Mier [11] for instance report that a supercomputer needed more than two weeks to analyze a 449179 beam model. A solution to avoid huge calculation times is a multiscale approach in which the discrete representation of trusses and beams at the microscale is used to characterize the mechanical constitutive behavior at the macroscale. Examples of such studies are those of Heyden [1] for cellulose fiber fluff, of Boisse et al. [17] for textile, and Mohr [18] for an ideal truss lattice. In the study of Stylianopoulos and Barocas [8], a collagen network is represented by trusses and coupled to the macroscale model via a computational homogenization scheme. These homogenization-based approaches, however, are not very appropriate to study localized phenomena, such as failure.

An alternative multiscale methodology that takes discrete lattice defects into account is to combine continuum models with lattice models. The lattice model is used in small regions of interest around the defects, whereas continuum models-discretized using the finite element (FE) method-are used for the remaining domain. This combined approach is widely used for atomic crystals (for instance by Xiao and Belytschko [19] and Fish et al. [20]) but it can only be used if the effective behavior of the lattice is adequately captured in a continuum model. Capturing the continuum equivalent behavior of the lattice, however, becomes more difficult as the amount of detail required in the lattice description increases. This occurs, for example, if plasticity is included [18], if the complexity of the lattice increases [12], or if beams are used instead of trusses, since such models also involve rotational degrees of freedom (DOFs) [16]. Even general phenomena such as an evolving microstructure due to fiber reorientation can lead to complex continuum models [21], while such phenomena are intrinsically included in the underlying lattice models. Another substantial difficulty of a combined continuum-lattice model approach is the coupling between both domains, which is far from trivial $[19,20]$.

An approach proposed for crystals which combines the best of both, is the quasicontinuum (QC) method [22,23]. In this method a number of representative atoms are defined and the positions of the remaining atoms are interpolated between them. For this purpose, the crystal is triangulated, where large interpolation triangles are used in uniformly deforming regions and the triangle size is reduced to the atomic spacing near features and events of interest. The equations which govern the remaining equilibrium equations, i.e. those associated with the representative atoms, are constructed by approximating the contributions of large numbers of atoms to the energy of the system via a so-called summation rule. The method exclusively relies on the lattice model and does not require the definition of an accompanying continuum model. The triangular interpolation regions are thus not traditional FEs (for the discretization of a continuum) but exist only to interpolate the lattice model. Furthermore, no continuum-atomistic coupling procedure is required, since only the lattice model is used. Different QC methods have been developed that all have their specific scope, advantages, and disadvantages [22-26]. Overviews are given by Miller and Tadmor [27] and Curtin and Miller [28].

Although the QC method was originally proposed by Tadmor et al. [22] for atomic crystals and applications, such as nanoindentation [23] and intergranular fracture [29], it offers appealing possibilities for structural lattice models incorporating trusses and beams. As a first step towards complex structural lattice models, this paper focuses on the development of the QC method for truss networks which can be regarded as a lattice model with a simplified discrete microstructure. The existing QC methods based on atomic lattices are therefore revisited with a new emphasis 
towards this truss network, which leads to a new QC method for these types of lattice models that also solves certain disadvantages of the classical QC methods.

The newly proposed QC method has the advantage that, similar to the cluster QC methods $[25,26]$, no internal interface is introduced. As a result, the fully resolved regions around lattice defects can remain small, thus limiting the global number of unknowns. The proposed QC method is therefore computationally efficient. However, unlike the existing approaches, no estimate of the total potential energy is made but the exact total potential energy is recovered. Although the method has been developed for a lattice model containing simple trusses, it can be used for general lattices with nearest neighbor interactions and, with some simple modifications, also for lattices with next-nearest neighbor interactions such as atomic lattices.

This paper aims to establish the basis for a QC method for lattice models of fibrous and heterogeneous materials. In particular, we consider a two-dimensional, regular linear elastic truss network, which acts as the discrete representation of a fictitious microstructure. Three different loading situations are considered to investigate the accuracy of the QC method for this lattice model. Uniform deformation is analyzed as a reference case, followed by bending as an example of a slowly fluctuating deformation. Finally, a truly multiscale example of a defect in an otherwise uniformly deforming lattice is modeled to investigate computational cost versus accuracy.

The outline of the paper is as follows. In Section 2 the new QC method based on the considered structural lattice model is introduced. In Section 3 results obtained for three examples are compared to direct simulations of the full lattice model and a semi-QC method without summation. Finally, conclusions are presented in Section 4.

\section{A QC APPROACH FOR LATTICE MODELS}

\subsection{Lattice model}

Structural lattice models are widely used to describe material behavior of fibrous and heterogeneous materials. Depending on characteristic properties of the discrete microstructures, trusses or beams (or both) are commonly used to model the discrete interactions. Rotational springs or trusses can also be used to include a rotational stiffness between discrete elements. Different discrete failure mechanisms may be included as well, e.g. fiber breakage in fibrous materials or contact failure between discrete particles in heterogeneous materials. Bond failure of fibrous materials may be modeled by sliding of the discrete elements.

Within the wide scope of structural lattice models, this paper aims to establish a QC methodology for a relatively simple lattice model in order to show the potential of the method for fibrous and heterogeneous materials. The considered reference lattice model is a two-dimensional X-braced linear elastic truss network as shown in Figure 1. This simple lattice model can be regarded as the discrete representation of a fictitious material. In it, each of the nodes, which are organized in a square pattern, is connected by trusses to its immediate neighbors in the horizontal, vertical, and diagonal sense. No truss nodes are present at the crossings of (only) the diagonal trusses.

To determine the equilibrium state of the (loaded) network, we minimize its total potential energy. Half of the potential energy of the truss between nodes $i$ and $j, E_{i j}$, is assigned to node $i$ and the other half to node $j$. The obtained nodal energy $E_{i}$ can be expressed as follows:

$$
E_{i}=\frac{1}{2} \sum_{j \in B_{i}} E_{i j}=\frac{1}{4} \sum_{j \in B_{i}} k_{i j}\left(\Delta L_{i j}\right)^{2}
$$

where the index $j$ refers to the neighboring truss nodes of node $i$, which form a subset $B_{i}$ of the index set $N=\{1, \ldots, n\}$ of the truss nodes of the lattice $\left(B_{i} \subseteq N\right)$. The potential energy $E_{i j}$ of a single truss element is expressed in terms of its stiffness $k_{i j}$ and the truss elongation $\Delta L_{i j}$. The fully non-linear elongation $\Delta L_{i j}$ is used here and therefore the total potential energy is non-convex. 


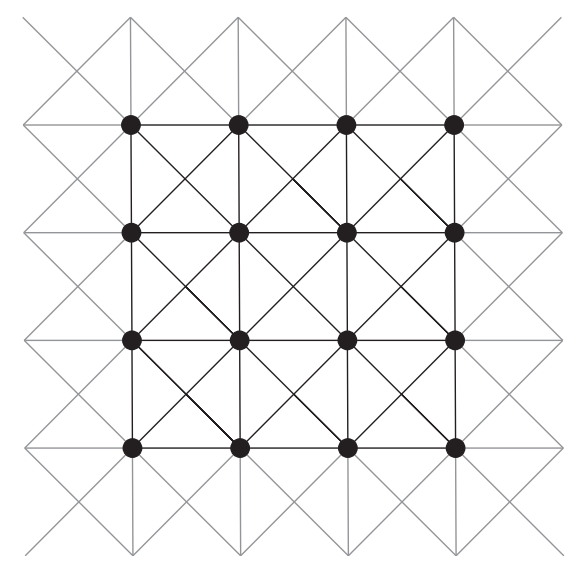

Figure 1. Schematic representation of nine unit cells (dark) of the considered two-dimensional, regular linear elastic truss network. The dots are nodes of the truss network.

Now that the nodal energies of all truss nodes of the lattice have been established according to Equation (1), the internal potential energy $E_{\text {int }}$ of the entire lattice can be obtained by summing all nodal potential energies of the entire lattice:

$$
E_{\text {int }}(\mathbf{u})=\sum_{i=1}^{n} E_{i}
$$

$E_{\text {int }}$ is a function of the displacement vectors of all $n$ truss nodes of the lattice. The components of all displacement vectors are collected in a column matrix $\mathbf{u}$ with length $n d$, where $d$ equals the number of spatial dimensions of the lattice.

The total potential energy $E_{\text {tot }}$ can now be expressed as a function of the internal potential energy $E_{\text {int }}$ and the external forces $\mathbf{f}_{\text {ext }}$ :

$$
E_{\mathrm{tot}}(\mathbf{u})=E_{\mathrm{int}}(\mathbf{u})-\mathbf{f}_{\mathrm{ext}}^{\mathrm{T}} \mathbf{u}
$$

The total potential energy can subsequently be minimized with respect to all DOFs, which are the displacement components present in column $\mathbf{u}$ :

$$
\min _{\mathbf{u}} E_{\mathrm{tot}}(\mathbf{u}) \text {. }
$$

The minimum of $E_{\text {tot }}$ can be determined using the classical variational principle, leading to

$$
\delta E_{\text {tot }}(\mathbf{u})=\delta \mathbf{u}^{\mathrm{T}} \mathbf{f}(\mathbf{u})=0 \quad \forall \delta \mathbf{u}
$$

where $\mathbf{f}(\mathbf{u})$ represents the column with conservative forces $\partial E_{\text {tot }} / \partial \mathbf{u}$ and has length $n d$. It can be assembled from contributions $\mathbf{f}_{i}$ by each node $i$ according to

$$
\mathbf{f}(\mathbf{u})=\sum_{i=1}^{n} \mathbf{f}_{i}(\mathbf{u})
$$

where

$$
\left(f_{i}\right)_{p}=\frac{\partial E_{i}}{\partial u_{p}}
$$

A standard Newton-Raphson procedure can be used to solve Equation (5):

$$
\delta \mathbf{u}^{\mathrm{T}}\left(\mathbf{f}\left(\mathbf{u}^{*}\right)+\mathbf{K}\left(\mathbf{u}^{*}\right) d \mathbf{u}\right)=0 \quad \forall \delta \mathbf{u}
$$


where $\mathbf{u}^{*}$ are the displacement components of the previous iteration and $d \mathbf{u}$ a correction to these displacements computed in the present iteration. The overall stiffness matrix is represented by $\mathbf{K}(\mathbf{u})$. Its elements are given by

$$
K_{p q}=\frac{\partial^{2} E_{\mathrm{tot}}}{\partial u_{p} \partial u_{q}}=\sum_{i=1}^{n} \frac{\partial^{2} E_{i}}{\partial u_{p} \partial u_{q}}
$$

where $p$ and $q$ run over all $n d$ displacement components in the column $\mathbf{u}$. It has size $n d \times n$ $d$ and is symmetric. It is again convenient to think of $\mathbf{K}$ consisting of contributions $\mathbf{K}_{i}$ by each node, i.e.

$$
\mathbf{K}=\sum_{i=1}^{n} \mathbf{K}_{i}
$$

where

$$
\left(K_{i}\right)_{p q}=\frac{\partial^{2} E_{i}}{\partial u_{p} \partial u_{q}} .
$$

After taking the essential boundary conditions and partioning the overall stiffness matrix, the remaining part of the stiffness matrix necessary to solve the system must be positive definite to obtain a locally stable configuration of the lattice.

A full lattice calculation is computationally inefficient for two reasons. First, it includes a large number of DOFs, which produces a large stiffness matrix-here with size $n d \times n d$. Solving the linear system in Equation (8) becomes computationally expensive, even if the Cholesky decomposition or an iterative solution method is applied. The second computational concern results from the fact that in order to construct the overall stiffness matrix, all $n$ truss nodes must be visited individually (see Equation (9)).

\subsection{Interpolation}

The QC method (proposed by Tadmor et al. [22]) was developed to overcome both of the above problems. First, interpolation is used to reduce the number of DOFs of the model. For this purpose the lattice is triangulated as shown in Figure 2. The interpolation relates the displacement vectors of truss nodes within each triangle to the displacement vectors of the three corner nodes of the triangle. As in QC methods the nodes of the triangles are placed on truss nodes and these truss nodes therefore represent the displacements of the truss nodes surrounding them, they are referred to as representative nodes or repnodes, in accordance with the term repatoms used in existing QC methods for atomic crystals. The interpolation constrains truss nodes within interpolation triangles and the displacements of the repnodes are the remaining DOFs. So far only linear interpolation has been used in the QC methods, for which nodes or atoms behave in an affine manner within the triangles.

In the QC method, the triangulation is done in such a way that the exact lattice model is recovered in regions where small details need to be fully resolved, while in other regions the lattice model is interpolated by coarsening the triangulation (see Figure 2). Regions require modeling with full accuracy if locally high strain gradients occur due to missing truss nodes, failure of trusses, stiffer trusses, or locally imposed deformation. To ensure that the exact lattice model is captured in these fully resolved regions, all truss nodes in fully resolved regions are repnodes (see Figure 2).

The set $R$ containing the $r$ repnodes is a subset of $N(R \subseteq N)$. Since $r \ll n$, the solution space is drastically reduced by the interpolation and therefore the solution will generally obtain a higher energy. The displacement components of all $n$ truss nodes can be expressed as a function of the displacement components of the $r$ repnodes, stored in column $\mathbf{u}_{r}$, as follows:

$$
\mathbf{u}=\boldsymbol{\Psi} \mathbf{u}_{r}
$$

where $\boldsymbol{\Psi}$ is the condensation matrix of size $n d \times r d$, containing the values of the interpolation functions evaluated in the nodal positions. Since now the total potential energy only depends on the displacements of the repnodes, the minimization of the total potential energy must be performed 


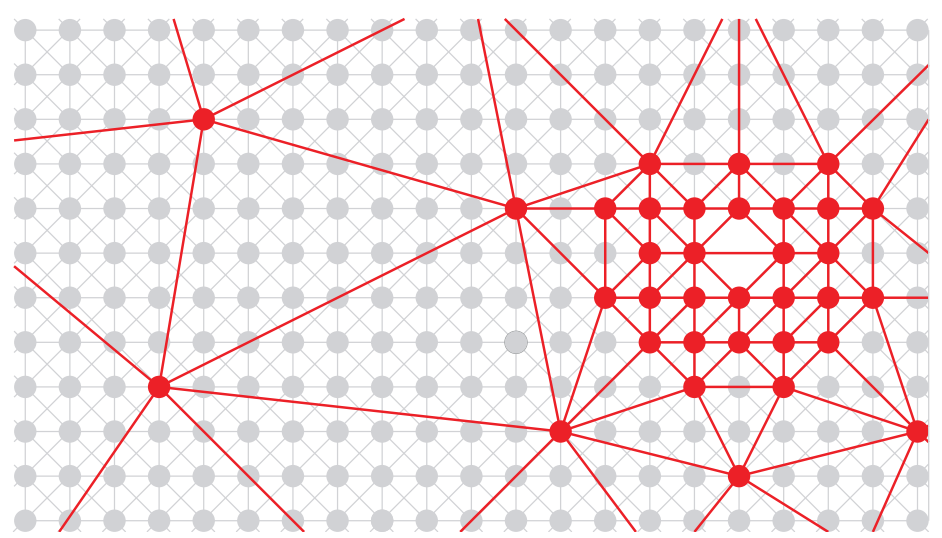

Figure 2. Schematic representation of a part of the two-dimensional X-braced truss lattice and a superimposed triangulation. The size of the triangles is reduced around the lattice defect so the exact lattice model is captured.

with respect to this reduced set of DOFs, i.e. the minimization problem (4) is replaced by

$$
\min _{\mathbf{u}_{r}} E_{\text {tot }}\left(\Psi \mathbf{u}_{r}\right) \text {. }
$$

This results in the following linearization instead of (8):

$$
\delta \mathbf{u}_{r}^{\mathrm{T}}\left(\boldsymbol{\Psi}^{\mathrm{T}} \mathbf{f}\left(\mathbf{u}^{*}\right)+\boldsymbol{\Psi}^{\mathrm{T}} \mathbf{K}\left(\mathbf{u}^{*}\right) \boldsymbol{\Psi} \mathrm{d} \mathbf{u}_{r}\right)=0 \quad \forall \delta \mathbf{u}_{r} .
$$

In Equation (14) $\boldsymbol{\Psi}^{\mathrm{T}} \mathbf{f}$ can be identified as the condensed force column $\mathbf{f}^{c}$ and $\boldsymbol{\Psi}^{\mathrm{T}} \mathbf{K} \boldsymbol{\Psi}$ the condensed stiffness matrix $\mathbf{K}^{c}$. The condensed force column and stiffness matrix are of size $r d \times 1$ and $r d \times r d$, respectively, and therefore considerably reduce the computational solution effort.

The interpolation used here for lattices has some similarities with FE methodologies for continua, in which FEs are used to discretize a continuum. For this reason characteristic features of conventional FE methods such as adaptivity can conveniently be adopted in QC methods. However, a substantial difference is that whereas in continua we are dealing with continuous displacement fields and the interpolation thus applies to every point in the domain, here the displacements of a discrete set of points govern the system and the interpolation thus also applies to a finite number of discrete points. Correspondingly, the energy of the system consists of a sum of pointwise contributions, whereas in the FE method integrals need to be evaluated.

Although the solution space is reduced at this stage, the assembly of the condensed force column and the condensed stiffness matrix remains a computationally expensive procedure, since all $n$ truss nodes must be visited for it:

$$
\begin{aligned}
\mathbf{f}^{c}\left(\mathbf{u}_{r}\right) & =\sum_{i=1}^{n} \boldsymbol{\Psi}^{\mathrm{T}} \mathbf{f}_{i}, \\
\mathbf{K}^{c}\left(\mathbf{u}_{r}\right) & =\sum_{i=1}^{n} \boldsymbol{\Psi}^{\mathrm{T}} \mathbf{K}_{i} \boldsymbol{\Psi} .
\end{aligned}
$$

Note that the nodal energy $E_{i}$, and thus the force column $\mathbf{f}_{i}$ and the stiffness matrix $\mathbf{K}_{i}$ in Equation (15) and (16), only depend on the displacements of truss node $i$ and its neighbors, which results in a large amount of zeros in the force column and stiffness matrix. Consequently, only a part of the condensation matrix $\boldsymbol{\Psi}$ is used for the matrix multiplications in Equations (15) and (16), allowing one to exploit the sparsity of the matrices in the numerical implementation of the method.

\subsection{Summation}

Although the number of DOFs and thus the size of stiffness matrix and right-hand side is substantially reduced by the interpolation, still all $n$ truss nodes of the lattice must be visited to assemble 
the force column and the stiffness matrix (see Equations (15) and (16)). To overcome this expensive assembly the QC method uses only a small number of $s$ truss nodes to approximate the total internal potential energy, and thus the force column and the stiffness matrix, instead of determining them via Equations (15) and (16). This operation has some similarity with the use of integration points to numerically integrate the stiffness matrix in the FE method. Here, however, we are dealing with a sum of discrete contributions, which are associated with discrete points in space. Consequently, the rules used to construct approximations for this sum are referred to as summation rules.

The truss nodes selected to sample the nodal energies of their surrounding truss nodes are referred to here as sampling nodes. They are gathered in index set $S \subseteq N$. Sampling nodes may coincide with the repnodes, as for instance in the node-based QC framework of Knap and Ortiz [25] in which the two sets are identical $(S=R)$.

The number of surrounding nodes that are represented by one sampling node must be carefully determined and constitutes the weight factor of the corresponding nodal energy. The assembly procedures of Equations (15) and (16) can now be rewritten as follows to obtain the condensed force column and stiffness matrix:

$$
\begin{aligned}
\mathbf{f}^{c, s}\left(\mathbf{u}_{r}\right) & =\sum_{i \in S} w_{i} \boldsymbol{\Psi}^{\mathrm{T}} \mathbf{f}_{i} \\
\mathbf{K}^{c, s}\left(\mathbf{u}_{r}\right) & =\sum_{i \in S} w_{i} \boldsymbol{\Psi}^{\mathrm{T}} \mathbf{K}_{i} \boldsymbol{\Psi}
\end{aligned}
$$

where the nodal energy of sampling node $i$ is used to approximate the nodal energies of $w_{i}$ nodes. The sizes of $\mathbf{f}_{i}, \mathbf{K}_{i}$, and $\boldsymbol{\Psi}$ remain $n d \times 1, n d \times n d$, and $n d \times r d$, respectively. To ensure that the computational burden is reduced, the number of sampling nodes must be substantially smaller than the total number of nodes, i.e. $s \ll n$.

The question arises which sampling nodes must be selected to establish an accurate estimate of the total potential energy (and thus the condensed force column and stiffness matrix). It is important that the selection of sampling nodes is in accordance with the triangulation. If the selection is poorly done an incorrect solution or even zero-energy modes may occur, as is shown in [25]. Different QC methods can be distinguished based on different selection procedures (summation rules): the localnonlocal QC method, the node-based QC variant and its generalization, the cluster QC method.

The local-nonlocal QC method is characterized by a different energy evaluation of the sampling atoms for the interpolated domain and the fully resolved domain. In the QC methods of Tadmor et al. [22], Miller et al. [29], Shenoy et al. [24], and Shimokawa et al. [30] all repatoms are selected as sampling atoms but the computation of the sampling atoms in the fully resolved region is nonlocal while it is local in the interpolated domain. In the fully resolved domain all atoms are repatoms and all repatoms are sampling atoms to ensure the accuracy of the exact lattice model. This part of the domain is called nonlocal because (atomic) interactions at finite distances are fully preserved.

In the interpolated region the repatoms are selected as sampling atoms as well. However, the site energies of these sampling atoms are determined as if the atoms were embedded in an infinitely large, perfect crystal. This is referred to as local QC, since the computation of sampling atoms is local, and is equivalent with homogenization towards a continuum according to the Cauchy-Born rule. The corresponding weight factor $w_{i}$ equals the number of atoms in the considered triangle and can be determined in various ways [24].

A major disadvantage of this summation rule is that an internal interface occurs between the fully resolved (nonlocal) regions and the interpolated (local) regions. This is caused by the fact that in the local QC method separation of scales cannot be guaranteed close to the fully resolved regions. As a consequence, non-physical forces (ghost forces) occur at the interface. The interface must therefore be located relatively far away from the phenomena (e.g. defects) in which one is interested. This leads to large fully resolved regions, i.e. a large number of repatoms and sampling atoms, and thus to a relatively large computational burden. Corrective procedures have been formulated to deal with the internal interface $[24,30]$, but they are not without disadvantages. Corrective forces at the interface are for instance modeled as dead loads and they are therefore assumed not to change [24]. 


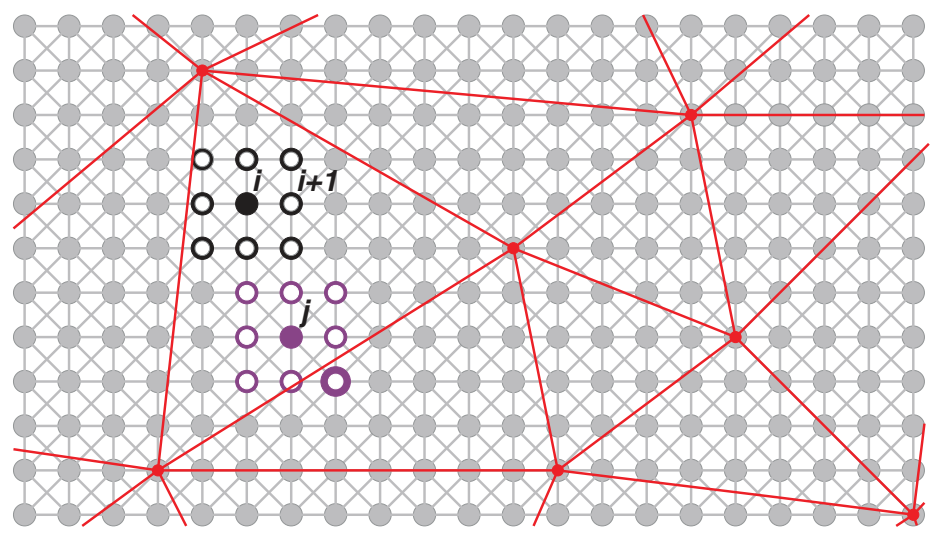

Figure 3. Schematic representation of a part of a two-dimensional regular truss network with a superimposed triangulation. The neighboring truss nodes of truss nodes $i$ and $j$ are visible as open circles. The bold neighboring truss node of truss node $j$ indicates that it is located in a different triangle than truss node $j$.

The QC method of Knap and Ortiz [25] has the major advantage above the local-nonlocal QC method that an internal interface between the interpolated and fully resolved regions does not occur. For this reason small fully resolved regions are in principle sufficient and thus the number of repatoms can remain small. The reason that no interface occurs is that the method uses the same sampling point (atom) computation for both domains and it does not use local computation of sampling atoms but merely nonlocal computation. If the repatoms are selected as sampling atoms, i.e. the node-based approach, this is dual to a Newton-Cotes integration scheme for continuum-based FE methods.

An important disadvantage of the node-based approach is that it may lead to zero-energy modes [25]. As a remedy for zero-energy modes, the use of clusters of sampling atoms around the repatoms has been suggested $[25,26]$. To retrieve the exact lattice model in fully refined domains, these clusters need to be truncated if they overlap.

A disadvantage of the cluster approach is the fact that the total potential energy is poorly estimated for any given cluster radius, as shown by Luskin and Ortner [31]. Moreover, the large number of sampling atoms and elaborate bookkeeping costs, partially caused by checks to determine if the clusters overlap, make the cluster QC method more inefficient than the local-nonlocal QC approach [27, 28].

Although the cluster QC method gives an inaccurate estimate of the total potential energy, it demonstrates that, in order to avoid an internal interface, one single selection procedure for the interpolated and the fully resolved domain is needed whereby the site energy of all sampling atoms is computed nonlocally (i.e. the Cauchy-Born rule is not applied).

\subsection{Novel summation rule}

In order to formulate a summation rule (i.e. a rule governing the selection and weight factor of sampling points) that satisfies the above conditions and nevertheless provides an accurate estimate, we first return to the interpolated system without summation (see Figure 3). This means that all truss nodes are visited, i.e. $S=N$, resulting in the exact potential energy of the interpolated system according to Equation (16). We will refer to this (expensive) method of constructing the reduced equations as the semi-QC method below.

As linear interpolation is used for the displacement vectors of the truss nodes within a triangle and since the nodal energies of the truss nodes only depend on their own displacements and the displacements of their nearest neighbors, the nodal energies of all truss nodes within a triangle are equal to each other if their nearest neighbors are located in the same triangle. An example of this is shown in Figure 3, in which the neighboring truss nodes of truss node $i$ are located in the same triangle as truss node $i$. As the neighboring truss nodes of truss node $i+1$ are also located in the 
same triangle (see Figure 3), the trusses associated with these nodes are deformed identically and their nodal energies are equal to each other. Note that, in terms of Equations (6) and (10) this also implies that $\mathbf{f}_{i}=\mathbf{f}_{i+1}$ and $\mathbf{K}_{i}=\mathbf{K}_{i+1}$. On the other hand, the nodal energy of truss node $j$ does not have to be equal to that of truss nodes $i$ and $i+1$ since one of the neighbors of truss node $j$ is located in another triangle than $j$ and therefore the nodal energy of truss node $j$ also depends on the deformation of the neighboring triangle.

In interpolated regions with large triangles, the majority of nodes have their neighbors in the same triangle. The energies of these nodes, which are all equal, may be poorly represented by the repnodes or clusters around them, as proposed in the cluster QC method [25, 26]. For this reason, the cluster QC method gives an inaccurate estimate of the potential energy. Even if the cluster radius is large enough so that every node is used in the summation $(S=N)$ and the improved method of Eidel and Stukowski [26] is used, the cluster summation rule determines the weight factors ( $w_{i}$ in Equations (17) and (18)) in such a way that the exact internal potential energy is not found (see also the study of Luskin and Ortner [31]).

Instead of sampling the energy at (or near) the repnodes, the above observations suggest a focus on the central regions of each triangle, where the nodal energy is constant, and therefore also the contributions $\mathbf{f}_{i}$ and $\mathbf{K}_{i}$ to the linearized equilibrium equations. According to the aforementioned analysis of the semi-QC approach, in which only interpolation is performed $(S=N)$, the total potential energy is not affected if only truss nodes with all their neighbors in the same triangle are summed. Therefore the new QC method uses only one of these nodes for sampling, i.e. only one of them is included in $S$ and its corresponding weight factor $w_{i}$ is set equal to the number of truss nodes within the triangle that have no neighbors in other triangles.

In practice we use the first node encountered which has all of its neighbors in the triangle. It is emphasized that this is an arbitrary choice and any other choice among the internal nodes would also give an exact result. An exact summation is obtained if all other truss nodes, that have one or more neighbors in another triangle, are taken into account individually. They can also be regarded as sampling nodes (i.e. they also belong to $S)$, with a weight factor equal to $1\left(w_{i}=1\right)$ since they 'estimate' only their own energy. We refer to these nodes as discretely modeled nodes. Because in this summation rule only truss nodes are eliminated that have the same energy as the corresponding sampling node, whereas all the nodes that have a potentially unique energy are individually taken into account, no summation error is introduced. The computed potential energy therefore equals the exact potential energy of the interpolated system and the condensed, summed force column and stiffness matrix in Equations (17) and (18) equal the condensed force column and stiffness matrix in Equations (15) and (16).

The proposed summation rule naturally avoids the introduction of an internal interface, since all (internal and discretely modeled) sampling nodes are computed nonlocally. This ensures that the discretely modeled sampling nodes interact with their actual neighbors in the adjacent triangles. For these nodes, it is important to emphasize that not only trusses crossing triangle edges are modeled but all trusses must be taken into account to correctly establish their nodal energy according to Equation (1).

As a result of the summation rule, bands of discretely modeled sampling nodes are obtained along edges of the interpolation triangles, while truss nodes inside the triangles are eliminated all but one, as can be seen in Figure 4. In small triangles, most notably in fully resolved regions, all truss nodes may have one or more neighboring truss nodes in other triangles and they are therefore all discretely modeled (see Figure 4). A smooth transition is achieved in this way towards fully resolved regions (where the exact lattice model is necessary) whereby the summation rule automatically uses fewer sampling nodes if fewer truss nodes become available in triangles with decreasing dimensions-cf. the shrinking of clusters in the cluster QC approach.

The bands of discretely sampled truss nodes along edges as shown in Figure 4 imply that still a relatively large number of truss nodes must be visited compared to e.g. the local QC method. For lattices with next-nearest neighbor interactions, such as atomic lattices, or three-dimensional lattices, these bands become wider and the computational efficiency may be too low. In follow-up work, we propose solutions to this concern, by no longer requiring that the estimated potential 


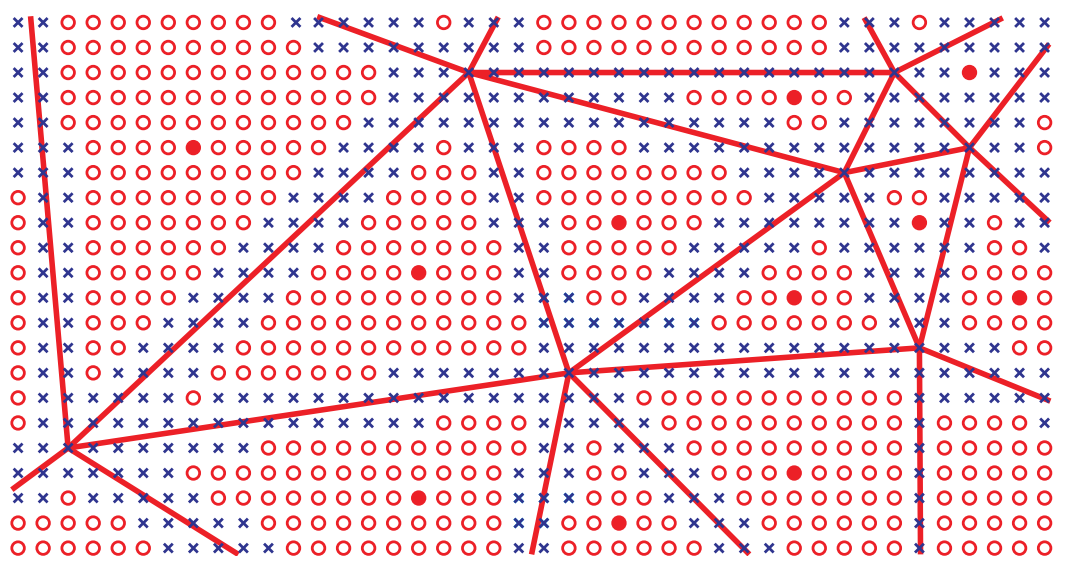

Figure 4. Sketch of the novel summation rule applied to a truss network. For clarity only the truss nodes are shown. Open circles represent truss nodes whose contributions are replaced by that of the internal sampling nodes (solid). Crosses represent discretely modeled nodes (for which $w_{i}=1$ ).

Table I. Algorithm of the proposed QC method.

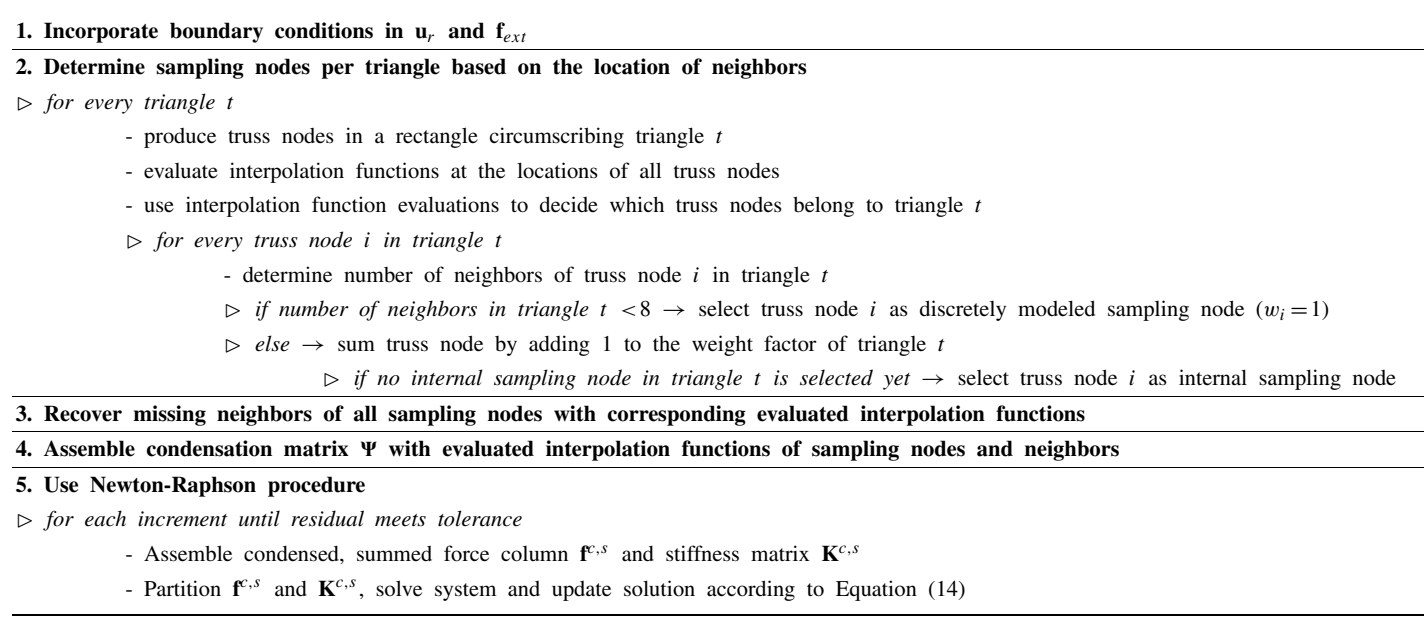

energy is exact, but accepting some error. However, for two-dimensional structural lattice models with only nearest neighbor interactions such as the example considered in this study, the computational cost of the exact method remains acceptable as will be shown in Section 3.

\subsection{Algorithm}

The proposed summation rule does not result in an internal interface, which leads to a simple and efficient algorithm. The algorithm for the new QC method is presented in Table I. The new summation, and thus the algorithm, is triangle-based and separately deals with each triangle. Therefore, the algorithm is relatively straightforward to implement and it can be parallelized with some minor adaptations to increase its efficiency.

The essential step of the summation rule is the second step in Table I, in which the location of the neighbors of every truss node of the lattice is used to determine whether it is selected as a sampling node and thus belongs to $S$. First the truss nodes of a particular triangle are selected in an efficient manner, for which the interpolation functions are evaluated at the lattice positions. It is advantageous to use the evaluated interpolation functions for this decision because they are also necessary in the fourth step of the algorithm. At this point all truss nodes of the considered triangle are known and by a simple count if a truss node has eight neighbors in this triangle it can 
be decided if the truss node must be selected as discretely modeled node and therefore must be assigned to set $S$ with $w_{i}=1$. Otherwise, the truss node is summed and one of the internal truss nodes is selected as sampling node with a corresponding weight factor that equals the number of summed truss nodes. After all truss nodes of the lattice model have been processed, the set $S$ of sampling nodes is established.

To correctly determine the nodal energy of all sampling nodes in a nonlocal manner, all neighboring nodes of all sampling nodes must be available for the nonlocal computation of the sampling nodes' site energies. Therefore, in the third step of the algorithm the neighbors that are not in $S$ are recovered.

In the fourth step the evaluated interpolation functions are assembled to one matrix $\boldsymbol{\Psi}$ that is used to condense the summed force column and stiffness matrix (see Equations (17) and (18)) in the Newton-Raphson procedure of the fifth step.

\section{PERFORMANCE STUDY}

In this section, the proposed QC method is applied to three different loading situations in order to assess its performance. First uniform tension is considered, by way of a patch test. This test demonstrates the accuracy of the calculated weight factors of the sampling truss nodes (see Figure 5(a)). Bending is the second considered deformation mode because it imposes gradual nonuniform deformation which triggers trusses that cross triangle edges to behave differently than trusses within triangles (Figure 5(b)). A case including a lattice defect is used to assess the accuracy of the proposed QC method for microscale lattice defects in a large-scale model (Figure 5(c)). Finally, the impact of the scale/size of the problem domain on the efficiency is examined by successively increasing the model size while keeping the defect constant.

A lattice model consisting of 70 by 50 unit cells (Figure 1) in horizontal and vertical direction, respectively, is used as a reference model. The stiffnesses of the horizontal and vertical trusses are equal and the stiffness of the diagonal trusses equals a factor $1 / \sqrt{2}$ times that of the horizontal and vertical trusses. The trusses along the four outer boundaries are given half the usual stiffness to ensure that a state of uniform deformation is recovered for uniform tension. The results are compared to the direct lattice simulation and to the semi-QC method, which gives the best achievable result for a given triangulation.

\subsection{Uniform tension}

Uniform deformation is simulated for six triangulations as presented in Figure 6 by applying $0.5 \%$ strain in horizontal direction to the right edge of the model. The model is free to contract in vertical direction. In the direct simulation (not shown) a perfectly uniform deformation is obtained and the site energies of all nodes in the interior of the model are therefore equal. The linear interpolation used in the QC method should be perfectly capable of capturing uniform deformation and should therefore not introduce any error. In Figure 6, it can be clearly seen that for large triangles the new summation rule leads to a relatively large reduction of the number of sampling nodes compared to small triangles, since in small triangles relatively many truss nodes have one or more neighbors in an adjacent triangle and are therefore selected as (discretely modeled) sampling nodes.

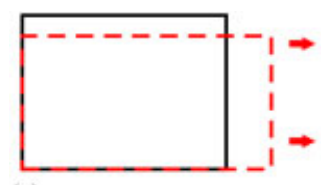

(a)

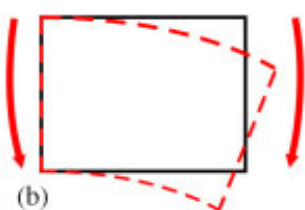

(b)

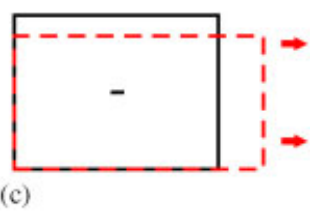

(c)

Figure 5. The three numerical test cases: (a) uniform tension; (b) bending; and (c) uniform tension with a missing truss in horizontal direction. 

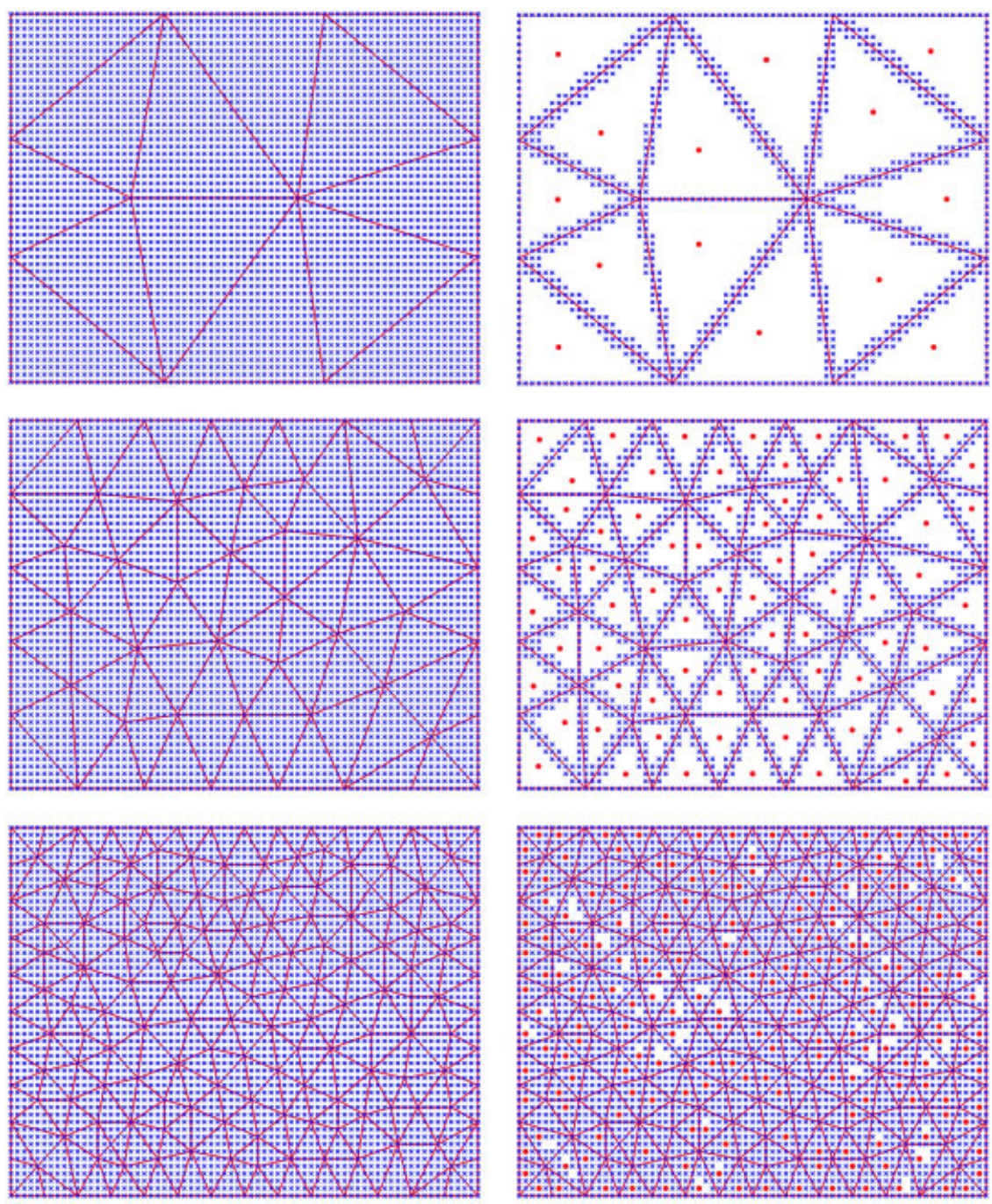

Figure 6. A coarse, intermediate, and fine triangulation are shown from top to bottom, with average triangle sizes of 250,49 , and 12 unit cells, respectively. On the left the semi-QC variant with solely interpolation is depicted (no summation) whereas on the right the new summation rule is applied. The full circles indicate internal sampling nodes that represent all other truss nodes in a triangle which have their neighbors in the same triangle. The crosses represent discretely modeled nodes.

The total internal energies for uniform deformation are presented in the left graph of Figure 7. The results for the proposed QC method equal the results of the direct simulation and the semi-QC result for all triangulations. This indicates that all weight factors are correctly determined in the new QC method, consistently with the selected sampling nodes. Although it may seem trivial that the exact result is obtained in this patch test, not all existing QC methods pass this test.

\subsection{Bending}

The same lattice and triangulations are used for bending, where the applied strain in horizontal direction increases linearly from $-0.5 \%$ at the bottom to $0.5 \%$ at the top of the truss network. Comparing the total internal energies of the proposed QC method and the exact lattice model in the right graph in Figure 7, it is clear that the coarse triangulation is not fine enough to predict the bending energy of the direct lattice simulation, which is expected for a linear interpolation-cf. the poor performance of linear FEs in bending. For the finer triangulations the total internal energies of the proposed QC method tend towards the full lattice result, i.e. towards $E / E_{d s}=1$, but the exact 

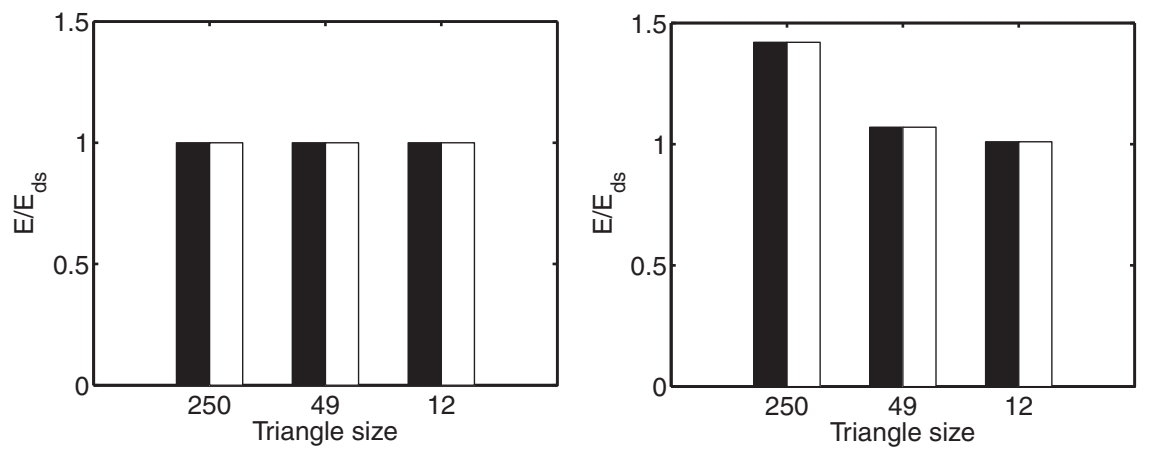

Figure 7. (Left) Computed energy in case of uniform deformation normalized by the energy of the direct simulation for different average triangle sizes in terms of unit cells. (Right) Bending energy normalized by the energy of the direct simulation for different average triangle sizes. Black bars: semi-QC method; white bars: the novel QC method.

internal energy is not reached. However, the energy computed by the proposed QC method does equal that of the semi-QC method, indicating that the summation remains optimal in the proposed QC method. The correct summation implies that not only intra-triangular interactions, i.e. the weight factors and corresponding sampling nodes, are exact but also inter-triangular interactions are adequately resolved.

\subsection{Single lattice defect}

To investigate the multiscale character of the QC method, an example with a horizontal lattice defect is studied. The same 70 by 50 unit cell lattice model is used, subjected to a uniform deformation at the boundary as in the patch test of Section 3.1. However, a horizontal lattice defect has been introduced by removing the right horizontal truss of the center truss node, as shown in Figure 5.

Six different triangulations as shown in Figure 8 are used to investigate the influence of the defect and the capability of the QC method to resolve it. Triangulations $a$ to $e$ in Figure 8 have fully resolved regions of $2 \times 2,4 \times 4,6 \times 6,8 \times 8$, and $10 \times 10$ lattice distances, respectively. The triangle size in the interpolated region doubles with every ring of triangles away from the fully resolved region. Triangulation $f$ has a fully resolved region of $4 \times 4$ lattice distances, like triangulation $b$, but the triangle size in the interpolated region doubles only after every second triangle.

In the left graph of Figure 9 the number of repnodes and sampling nodes relative to the 3621 truss nodes of the complete lattice are shown for each triangulation. The relative number of repnodes is smaller than $5 \%$ for all triangulations but the relative number of sampling nodes reaches almost $70 \%$ for triangulation $f$. The reason for the large number of sampling nodes is that a small model is considered here; for large models the relative number of sampling nodes is substantially smaller (see Section 3.4). The difference in number of repnodes and sampling nodes between triangulation $a$ and $b$ is relatively large compared to the differences between triangulations $b$ and $c, c$ and $d$, and $d$ and $e$. This is due to the degree of refinement of the triangles in the interpolated region, which is clearly less complex for triangulation $a$ compared to triangulations $b$ to $e$. The number of sampling nodes for triangulations $b$ to $e$ increases similarly as the number of repnodes. This is caused by the fact that only the fully resolved region is enlarged while the refinement of the interpolated region remains almost the same (i.e. every extra repnode is also a sampling node). Comparing triangulation $b$ to triangulation $f$, it is clear that the number of sampling nodes is substantially larger for triangulation $f$ while the increase of the number of repnodes is small. The reason for this is the fact that the fully resolved regions are similar but the interpolated region of triangulation $f$ is more refined. Therefore more additional sampling nodes are created than repnodes.

Instead of focussing on the total internal energies-which hardly differ due to the presence of only one lattice defect-it is more interesting to establish if the proposed QC method predicts the displacements in the region around the lattice defect correctly. The horizontal components of the 


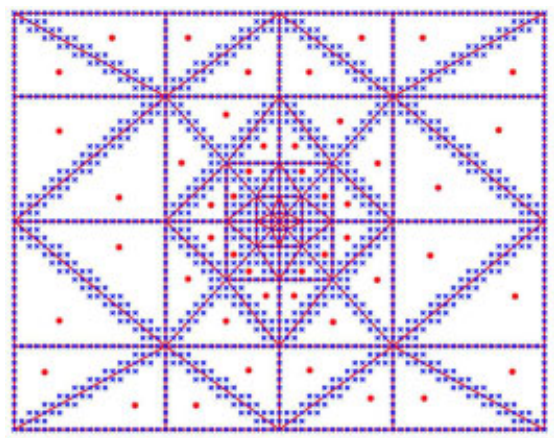

(a)

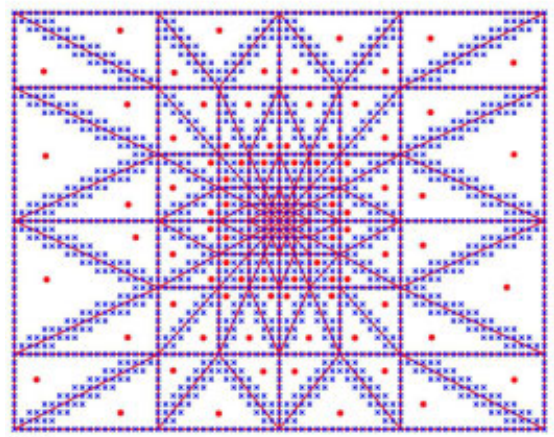

(b)

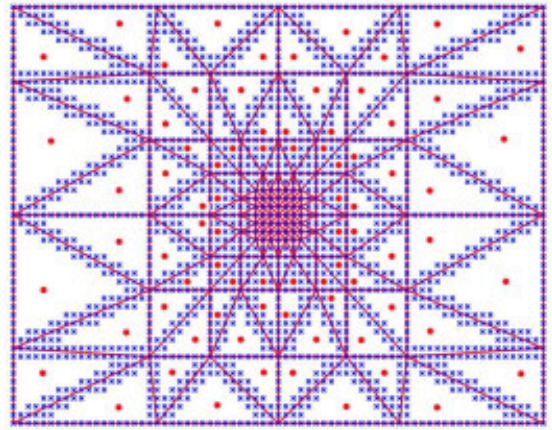

(c)

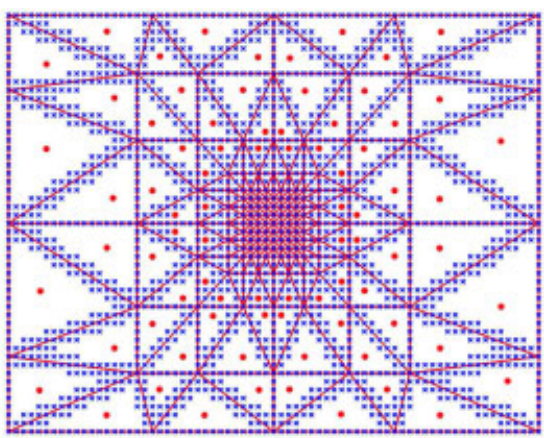

(d)

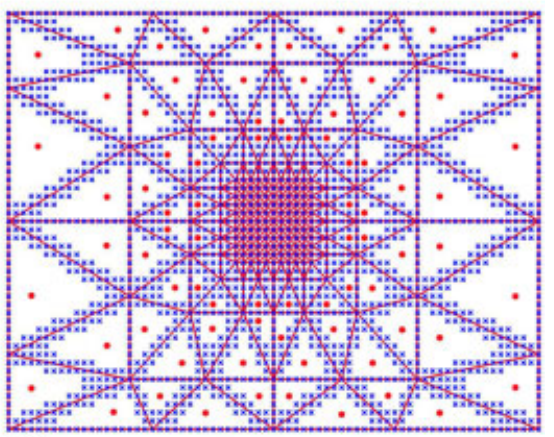

(e)

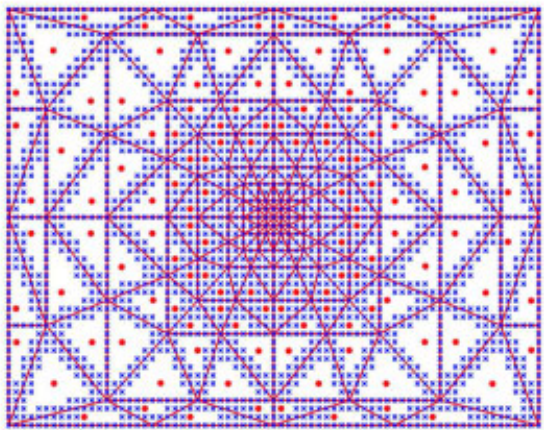

(f)

Figure 8. Six different triangulations to investigate the influence of the lattice defect. Full circles represent internal sampling nodes that represent all other truss nodes within one triangle that have all neighbors inside that triangle and crosses symbolize discretely modeled nodes.

displacement vectors of the truss nodes clearly show the fluctuation field caused by the defect, particularly if the displacements of the perfect lattice are first subtracted according to:

$$
u_{x}^{\mathrm{rel}}\left(\vec{x}_{i}\right)=u_{x}\left(\vec{x}_{i}\right)-E_{x x} x_{i}
$$

where the relative horizontal component of the displacement vector is denoted by $u_{x}^{\text {rel }}$ and $E_{x x}$ is the applied overall strain in $x$-direction.

For triangulation $c$ the relative horizontal displacements around the lattice defect as computed with the proposed QC method are presented in Figure 10. The defect causes two peaks at the two ends of the missing truss. Only the displacements in regions to the left and right of the lattice defect are influenced significantly while in the regions above and below the defect the relative displacement needs only a couple of lattice spacings to decay to zero.

The results of the proposed QC method are only compared to those of the direct lattice simulation since it is now clear that also for true multiscale models the results of the proposed QC method equal those of the semi-QC approach (not shown here). The maximum error of the fluctuation field 

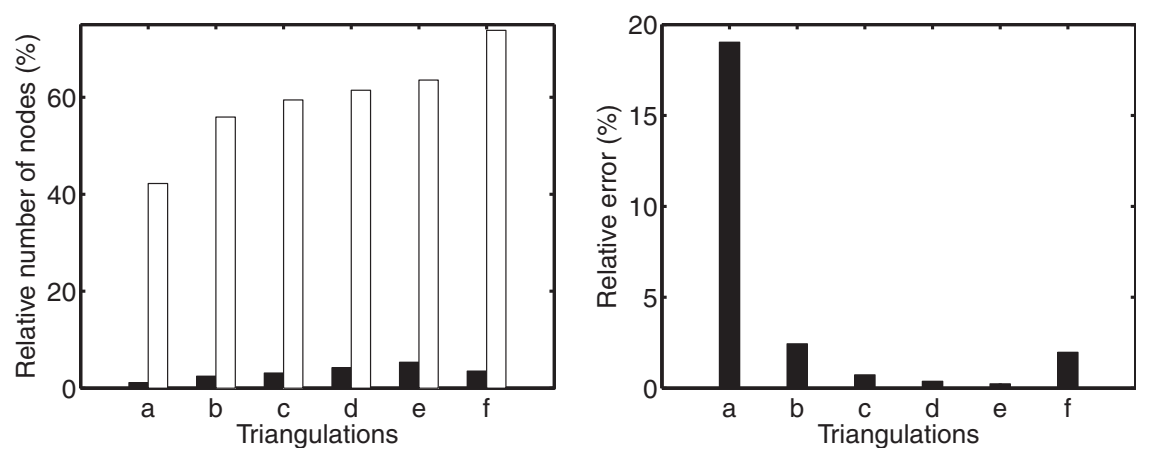

Figure 9. (Left) Black bars: the relative number of repnodes for the six triangulations; white bars: the relative number of sampling nodes. (Right) The maximum error of the relative displacements of the two truss nodes where the lattice defect is introduced.

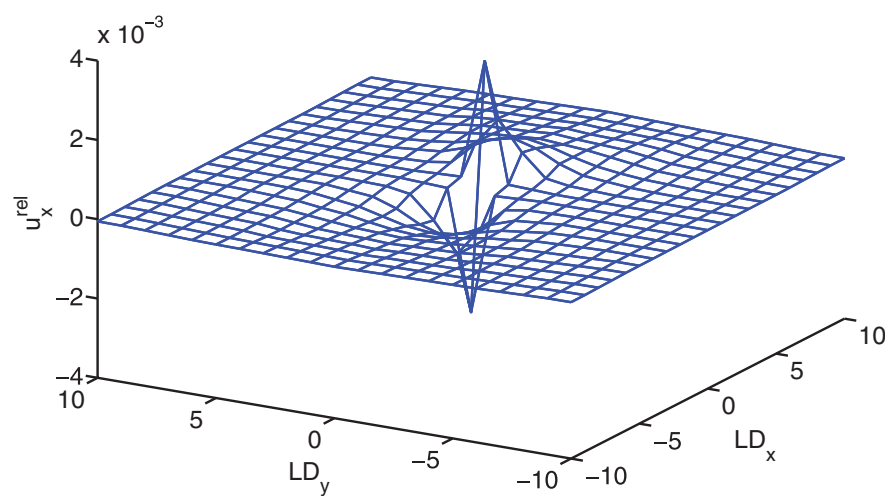

Figure 10. The relative horizontal displacements of the truss nodes, $u_{x}^{\text {rel }}$, for triangulation $c$ around the horizontal lattice defect as a function of the lattice distance in horizontal direction and vertical direction, denoted by $L D_{x}$ and $L D_{y}$, respectively.

of Figure 10 of the two truss nodes where the peaks occur is considered for this comparison. It is normalized by the maximum relative displacement in the direct simulation. Note that the defined error is a sensitive error measure since the normalization involves the displacements relative to the uniformly deforming lattice.

The maximum errors for the six triangulations are shown in the right graph of Figure 9. The error of triangulation $a$ is large since the missing truss is located at the edge of the interpolated region due to the fully resolved region of only $2 \times 2$ lattice spacings. If the distance between the lattice defect and the interpolated region is increased, the error is substantially reduced. For triangulation $b$, which has a fully resolved region of $4 \times 4$ lattice spacings, the relative error is reduced by a factor of 10. If the fully resolved region is further increased, the relative error decreases as well but by a smaller factor. For triangulation $c$, which has a fully resolved region of $6 \times 6$ lattice spacings, the error is less than $1 \%$, indicating that the proposed QC method gives an accurate result for a rather small fully resolved region.

Comparing triangulation $b$ and triangulation $f$, it is visible in the right graph of Figure 9 that a more refined interpolated region-as in triangulation $f$-only leads to a marginal reduction in the error. Since the number of repnodes and the number of sampling nodes increases substantially, it is more efficient to increase the size of the fully resolved region in order to reduce the error. Note, however, that this analysis is performed for uniform deformation while other loading situations might require a refined interpolated region instead of a larger fully resolved domain. 

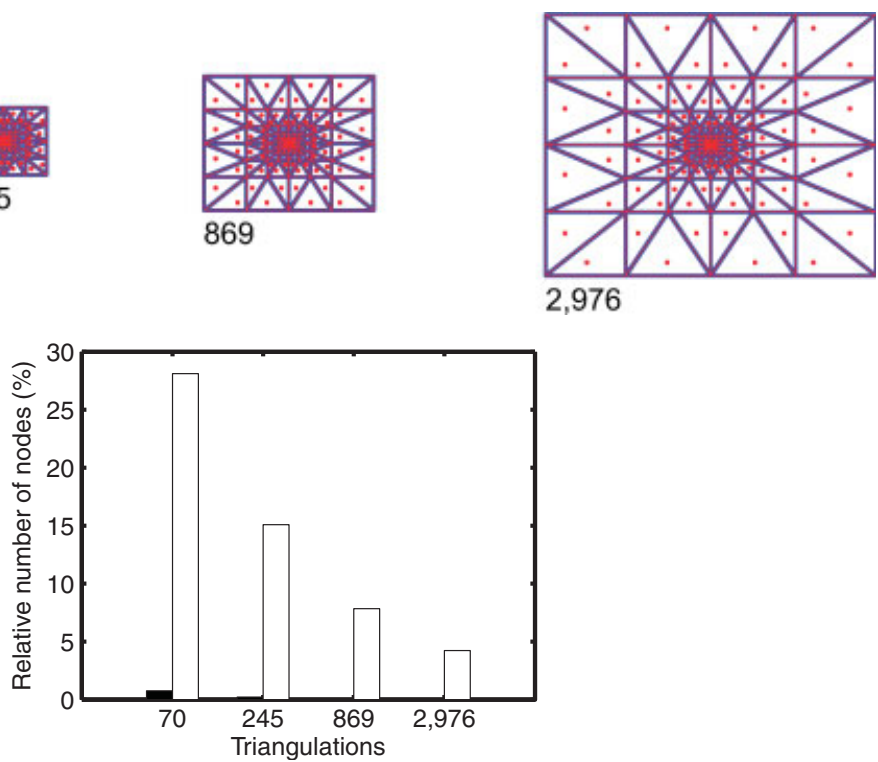

Figure 11. (Top) Four triangulations with fully resolved regions of $6 \times 6$ lattice spacings and average triangle sizes of 70, 245, 869, and 2976 unit cells. Every consecutive triangulation has an extra ring of triangles in the interpolated domain. (Bottom) Black bars: the relative number of repnodes for the four triangulations; white bars: the relative number of sampling nodes.

\subsection{Influence of model size}

The computational burden of the six models in Figure 8 is substantially reduced by the QC method compared to the direct lattice calculation. Especially the number of DOFs (displacement components of the repnodes) is reduced, while the obtained solution is almost identical to the direct lattice calculation. To ensure that the summation is exact, the number of selected sampling nodes remains rather high in the problem studied above (see the left diagram in Figure 9). However, for larger (physically relevant) models the number of sampling nodes is also reduced since in larger problems the interpolated regions comprise a relatively large part of the domain. This is illustrated in Figure 11, in which the size of the model has been increased while keeping the lattice spacing and defect size constant. This implies that the scale separation between the lattice and model is increased. The four triangulations shown have fully resolved regions of $6 \times 6$ lattice spacings and different sizes of interpolated regions around them. As can be seen in the graph in Figure 11 the number of repnodes and sampling nodes relative to the total number of truss nodes decreases for an increasing problem size and the efficiency of the QC method thus improves for larger scale separations between the fully resolved regions of interest and the complete model.

\section{CONCLUSION}

The QC method has so far only been used to reduce the computational cost of atomistic simulations [22-27, 29,30]. In this paper it is demonstrated that QC approaches also have a potential for structural lattice models containing discrete elements, such as trusses and beams. The high accuracy of the QC method proposed for structural lattice models has been illustrated in this paper by comparing the QC results to those of direct lattice calculations for a regular truss network. Furthermore, the large reduction of the number of DOFs, achieved by interpolation, and the fact that only a selective part of the truss nodes of the lattice model, so-called sampling nodes, has to be visited to construct the total internal potential energy, ensure that the QC method is markedly more efficient than direct simulations. 
The main reasons that a QC-type method is attractive for structural lattice models based on fibrous and heterogeneous materials are twofold. First, only a simple lattice model is needed in a QC approach. The method operates directly on this discrete model and no equivalent continuum model is required. This is an advantage because phenomena such as large rotations, re-orientation, anisotropy, bond fracture, sliding of nodes, and fracture of the discrete elements are difficult to capture in continuum models.

Second, if a fully resolved domain must be incorporated to investigate local events, no special coupling between the interpolated (continuum) domain and the fully resolved (discrete) domain is necessary. This is an advantage since such a coupling often invokes additional assumptions. Most QC methods use a coupling procedure to avoid interface problems between both domains but in this paper a $\mathrm{QC}$ variant is proposed that does not require such a coupling.

The QC method proposed here for structural lattices is the only QC method that determines the potential energy exactly while avoiding an internal interface between the interpolated and fully resolved domains. It is based on a clear understanding of how the total potential energy depends on the interpolation and how this should be reflected in the summation. This means that no summation error is introduced and the only occurring error is due to the interpolation.

The QC method has been evaluated for a relatively simple truss network which has a single lattice defect. For a relatively small fully resolved region (and thus a high computational efficiency) errors of less than $1 \%$ compared with the direct simulation have been observed. The same accuracy can be expected in case the damage progresses by subsequent removal of trusses. However, in this case the interpolation must be modified adaptively to ensure that the failure of trusses always takes place well within the fully resolved domain and the selection of sampling nodes must be updated accordingly. The damage progression is then completely dependent on the lattice model and independent of the triangulation since the triangle size in the fully resolved region is fixed to the lattice spacing and no failure of trusses occurs in the interpolated domain. The fact that the triangle size used in the interpolation has a natural minimum (corresponding to the fully resolved case) ensures that no pathological localization and mesh dependency occur upon refinement of the interpolation. In the limit of a fully resolved lattice model, the width of the process zone is set by the (physical) discrete lattice spacing.

Although the examples treated here are two-dimensional, the extension of the QC methodology to three-dimensional lattice models is straightforward. However, a concern may be that the computational efficiency of the summation rule used here is reduced. The reason for this is that tetrahedra contain relatively more nodes that have one or more neighbors in another tetrahedron, which are all taken into account discretely, than triangles. This is even more of a concern if lattice models with next-to-nearest neighbor interactions are considered, such as atomistic models. Modifications of the summation rule which deal with this issue will be discussed in a forthcoming publication.

Further research will also focus on applying the QC method to beam lattices, which contain next to translational DOFs also rotational DOFs. This combination of displacements and rotations may require a more advanced interpolation, akin to shell formulations in the FE method, which preserves consistency of the two sets of DOFs. Interestingly, such an approach would lead to an enriched QC on a natural discrete basis, and would allow us to make contact with Cosserat-type of continuum formulations [32].

\section{ACKNOWLEDGEMENTS}

This research is supported by the Dutch Technology Foundation STW, which is the applied science division of NWO, and the Technology Programme of the Ministry of Economic Affairs under Project Nr. 10104.

\section{REFERENCES}

1. Heyden S. Network Modelling for the Evaluation of Mechanical Properties of Cellulose Fibre Fluff. Lund University: Lund, Sweden, 2000.

2. Arnoux PJ, Bonnoit J, Chabrand P, Jean M, Pithioux M. Numerical damage models using a structural approach: application in bones and ligaments. The European Physical Journal of Applied Physics 2002; 17:65-73. 
3. Bronkhorst CA. Modelling paper as a two-dimensional elastic-plastic stochastic network. International Journal of Solids and Structures 2003; 40:5441-5454.

4. Clyne TW, Markaki AE, Tan JC. Mechanical and magnetic properties of metal fibre networks, with and without a polymeric matrix. Composites Science and Technology 2005; 65:2492-2499.

5. Potluri P, Manan A. Mechanics of non-orthogonally interlaced textile composites. Composites: Part A 2007; 38:1216-1226.

6. Lomov SV, Ivanov DS, Verpoest S, Zako M, Kurashiki T, Nakai H, Hirosawa S. Meso-FE modelling of textile composites: road map, data flow and algorithms. Composite Science and Technology 2007; 67:1870-1891.

7. Ben Boubaker B, Haussy B, Ganghoffer JF. Discrete models of woven structures. Macroscopic approach. Composites: Part B 2007; 38:498-505.

8. Stylianopoulos T, Barocas VH. Volume-averaging theory for the study of the mechanics of collagen networks. Computer Methods in Applied Mechanics and Engineering 2007; 196:2981-2990.

9. Zohdi TI. A computational framework of network modeling of fibrous biological tissue deformation and rupture. Computer Methods in Applied Mechanics and Engineering 2007; 196:2972-2980.

10. Hatami-Marbini H, Picu RC. An eigenstrain formulation for the prediction of elastic moduli of defective fiber networks. European Journal of Mechanics A/Solids 2009; 38:305-316.

11. Lilliu G, Van Mier JGM. On the relative use of micro-mechanical lattice analysis of 3-phase particle composites. Engineering Fracture Mechanics 2006; 74:1174-1189.

12. Cusatis G, Bazant ZP, Cedolin LC. Confinement-shear lattice model for concrete damage in tension and compression: 1. Theory. Journal of Engineering Mechanics 2003; 129:1439-1448.

13. Ostoja-Starzewski M, Wang G. Particle modeling of random crack patterns in epoxy plates. Probabilistic Engineering Mechanics 2006; 21:267-275.

14. Rinaldi A, Krajcinovic D, Peralta P, Lai YC. Lattice models of polycrystalline microstructures: a quantitative approach. Mechanics of Materials 2008; 40:17-36.

15. Kim H, Buttlar WG. Discrete fracture modeling of asphalt concrete. International Journal of Solids and Structures 2009; 46:2593-2604.

16. Ostoja-Starzewski M. Lattice models in micromechanics. Applied Mechanics Reviews 2002; 55:35-60.

17. Boisse P, Gasser A, Hivet G. Analyses of fabric tensile behaviour: determination of the biaxial tension-strain surfaces and their use in forming simulations. Composites: Part A 2001; 32:1395-1414.

18. Mohr D. Mechanism-based multi-surface plasticity model for ideal truss lattice models. International Journal of Solids and Structures 2005; 42:3235-3260.

19. Xiao BK, Belytschko T. A bridging domain method for coupling continua with molecular dynamics. Computer Methods in Applied Mechanics and Engineering 2004; 193:1645-1669.

20. Fish J, Nuggehally MA, Shephard MS, Picu CR, Badia S, Parks ML, Gunzburger M. Concurrent AtC coupling based on a blend of the continuum stress and the atomistic force. Computer Methods in Applied Mechanics and Engineering 2007; 196:4548-4560.

21. Peng XQ, Cao J. A continuum mechanics-based non-orthogonal constitutive model for woven composite fabrics. Composites Part A 2005; 36:859-874.

22. Tadmor EB, Philips R, Ortiz M. Quasicontinuum analysis of defects in solids. Philosophical Magazine A 1996; 73:1529-1563.

23. Tadmor EB, Ortiz M, Philips R. Mixed atomistics and continuum models of deformation in solids. Langmuir 1996; 12:4529-4534.

24. Shenoy VB, Miller R, Tadmor EB, Rodney D, Phillips R, Ortiz M. An adaptive finite element approach to atomistic scale mechanics - the quasicontinuum method. Journal of the Mechanics and Physics of Solids 1999; 47:611-642.

25. Knap J, Ortiz M. An analysis of the quasicontinuum method. Journal of the Mechanics and Physics of Solids 2001; 49:1899-1923.

26. Eidel B, Stukowski A. A variational form of the quasicontinuum method based on energy sampling in clusters. Journal of the Mechanics and Physics of Solids 2009; 57:87-108.

27. Miller RE, Tadmor EB. The quasicontinuum method: overview, applications and current directions. Journal of Computer-Aided Materials Design 2002; 9:203-239.

28. Curtin WA, Miller RE. Atomistic/continuum coupling in computational materials science. Modelling and Simulation in Materials Science and Engineering 2003; 11:33-68.

29. Miller R, Ortiz M, Phillips R, Shenoy V, Tadmor EB. Quasicontinuum models of fracture and plasticity. Engineering Fracture Mechanics 1998; 61:427-444.

30. Shimokawa T, Mortensen JJ, Schiøtz J, Jacobsen KW. Matching conditions in the quasicontinuum method: removal of the error introduced at the interface between the coarse-grained and fully atomistic region. Physical Review B 2004; 69:214104.

31. Luskin M, Ortner C. An analysis of node-based cluster summation rules in the quasicontinuum method. SIAM Journal on Numerical Analysis 2009; 47:3070-3086.

32. Casolo S. Macroscopic modelling of structured materials: relationship between orthotropic Cosserat continuum and rigid elements. International Journal of Solids and Structures 2006; 43:475-496. 